\title{
Magnetic field and electric currents in the vicinity of polar cusps as inferred from Polar and Cluster data
}

\author{
N. A. Tsyganenko \\ Institute of Physics, Saint-Petersburg State University, Saint-Petersburg 198504, Russia
}

Received: 1 January 2009 - Accepted: 27 February 2009 - Published: 2 April 2009

\begin{abstract}
A detailed statistical study of the magnetic structure of the dayside polar cusps is presented, based on multiyear sets of magnetometer data of Polar and Cluster spacecraft, taken in 1996-2006 and 2001-2007, respectively. Thanks to the dense data coverage in both Northern and Southern Hemispheres, the analysis spanned nearly the entire length of the cusps, from low altitudes to the cusp "throat" and the magnetosheath. Subsets of data falling inside the polar cusp "funnels" were selected with the help of TS05 and IGRF magnetic field models, taking into account the dipole tilt and the solar wind/IMF conditions. The selection funnels were shifted within $\pm 10^{\circ}$ of SM latitude around the model cusp location, and linear regression parameters were calculated for each sliding subset, further divided into 10 bins of distance in the range $2 \leq R \leq 12 R_{E}$, with the following results. (1) Diamagnetic depression, caused by the penetrated magnetosheath plasma, becomes first visible at $R \sim 4-5 R_{E}$, rapidly deepens with growing $R$, peaks at $R \sim 6-9 R_{E}$, and then partially subsides and widens in latitude at the cusp's outer end. (2) The depression peak is systematically shifted poleward (by $\sim 2^{\circ}$ of the footpoint latitude) with respect to the model cusp field line, passing through the $\min \{|\boldsymbol{B}|\}$ point at the magnetopause. (3) At all radial distances, clear and distinct peaks of the correlation between the local $B_{y}$ and $B_{y}^{(\mathrm{IMF})}$ and of the corresponding proportionality coefficient are observed. A remarkably regular variation of that coefficient with $R$ quantitatively confirms the field-aligned geometry of the cusp currents associated with the IMF $B_{y}$, found in earlier observations.
\end{abstract}

Keywords. Magnetospheric physics (Current systems; Magnetopause, cusp, and boundary layers; Magnetospheric configuration and dynamics)

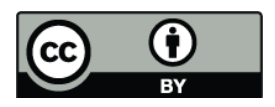

Correspondence to: N. A. Tsyganenko (nikolai.tsyganenko@gmail.com)

\section{Motivation of this work}

Polar cusps are an essential feature of the planetary magnetospheres. Their existence is a natural consequence of (1) the shielding effect of the incoming flow of highlyconducting solar wind and (2) conservation of the magnetic flux $(\nabla \cdot \boldsymbol{B}=0)$, which requires that the field lines cannot begin or end at any point of space, but can only diverge/converge and form null points or lines near the superconducting boundaries. The unique properties of the polar cusps were envisioned well before the beginning of the space age in a seminal work by Chapman and Ferraro (1931), in which a possibility of direct penetration of the solar plasma inside the magnetosphere was pointed out. Owing to the presence of the injected plasma in the cusps, one should expect a diamagnetic field depression to spread deep into the magnetosphere, in addition to the local vacuum-type depression near the magnetopause, associated with the divergence of the magnetic field lines near the cusp "throat". As shown in detail in our earlier work based on the first two years of Polar observations (Tsyganenko and Russell, 1999, referred henceforth as TR99) such depressions do indeed exist and, typically, can get as deep as $\sim-50 \mathrm{nT}$.

During the last decade, an ample amount of new data was provided by the Polar magnetic field experiment, now spanning virtually the entire magnetosphere within $R \leq 9 R_{E}$, thanks to the secular southward shift of the line of apsides of the spacecraft orbit. Another valuable new asset is the database of Cluster magnetic field observations made since its launch in 2000, covering the high-latitude magnetosphere at intermediate and large distances well beyond the apogee of Polar and thus offering an opportunity for more comprehensive studies of the outer cusps (e.g., Zhang et al., 2007). Figure 1 shows the coverage of the near magnetosphere by orbits of these two spacecraft, demonstrating the advantages of their high inclination with respect to the monitoring of the polar cusps.

Published by Copernicus Publications on behalf of the European Geosciences Union. 

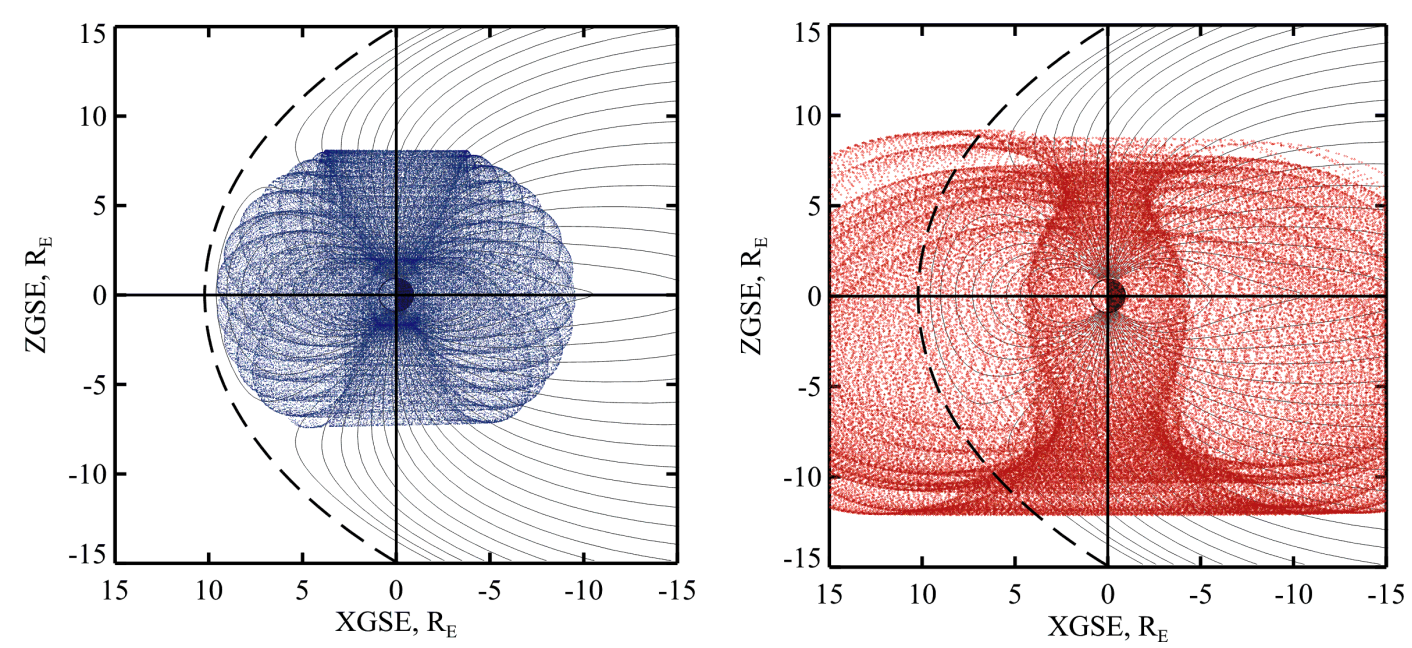

Fig. 1. Illustrating the coverage of the near magnetosphere $\left(X>-15 R_{E}\right)$ by observations of Polar (1996-2006; left panel) and Cluster (2001-2007; right panel). A typical magnetic field line configuration and the location of a model magnetopause are also shown.

The availability of such a wealth of data presents an attractive opportunity for further detailed studies. This paper addresses two characteristic features of the polar cusps: (1) the diamagnetic depression and (2) the effect of the IMF $B_{y}$ "penetration". The essence of the latter phenomenon is illustrated in Figs. 2 and 3, showing two examples of the polar cusp crossing by Cluster on 28 August 2001 and 4 September 2001. Three middle panels display the variation of three GSM components of the observed field (external part only) along with the predicted T96 model field near the spacecraft perigee at $\sim 4 R_{E}$ (Cluster's position in GSM coordinates is shown in the top panel). While there is a reasonable overall agreement between the observed and model $B_{x}$ and $B_{z}$ components (2nd and 4th panels from top), one immediately notices two large and sharp spikes of the observed $B_{y}$, not reproduced by the model. The spikes are negative in the first example and positive in the second one, matching in both cases the polarity of the concurrent $B_{y}^{(\mathrm{IMF})}$, shown in the bottom panel by green dots. Similar spikes can be observed in virtually all near-noon perigee passes of Cluster (occurring in August-September every year), and in each case there is a clear match of their polarity with that of $B_{y}^{(\mathrm{IMF})}$, which can be interpreted as a "penetration" of the IMF into the cusps.

In fact, this is a well-known phenomenon, associated with the field-aligned currents in the vicinity of the cusps. It was first described in the work by McDiarmid et al. (1979), followed by studies of Erlandson et al. (1988), Taguchi et al. (1993), and Zhou et al. (2000). The goal of the present work is to systematically explore the IMF $B_{y}$ effects and analyze in detail the near-noon cusp currents in the entire range of radial distances, in both Northern and Southern Hemispheres.

\section{Data}

In this study we used 1-min and 5-min average vector magnetic field data of Polar and Cluster (\#3) spacecraft, taken during the periods from 21 March 1996 through 31 December 2006, and from 1 February 2001 through 31 December 2007, respectively. Details of the magnetic field experiments onboard Polar and Cluster were described elsewhere (Russell et al., 1995; Balogh et al., 1997) and will not be addressed here. The time resolution of the data was chosen on the basis of an order-of-magnitude estimate of the duration of spacecraft crossing of the cusp field line tube, which depends on both its north-south thickness (varying roughly as $\sim R^{3 / 2}$ ) and on the local speed of the spacecraft. Because of the relatively high perigee of Cluster (geocentric $R_{p} \sim 4.3 R_{E}$ ) in comparison with that of Polar $\left(R_{p} \sim 1.7-1.9 R_{E}\right)$, all data of Cluster were used with 5-min resolution, while the Polar data were divided into two subsets: with $R \leq 5 R_{E}$ and $R>5 R_{E}$, in which the corresponding time resolutions were chosen equal to 1 and $5 \mathrm{~min}$, respectively. Due to much higher apogee of Cluster (hence, relatively short time spent in the vicinity of the cusps) and its significantly shorter total observation period in comparison with Polar, the overall percentage of Cluster data, measured by the number of records, is only $8.6 \%$, though in terms of the total observation time that estimate nearly doubles to $17.3 \%$.

As described in more detail in the next section, the adopted procedure of analyzing the data essentially depends on the possibility to accurately estimate the expected latitudinal position of both northern and southern cusps, which is quite sensitive to the concurrent conditions in the interplanetary medium, in particular, to the solar wind pressure and the orientation and magnitude of the IMF. In this work we used the OMNI source of 5-min average solar wind and IMF data, reduced to the subsolar bow shock position. The data 

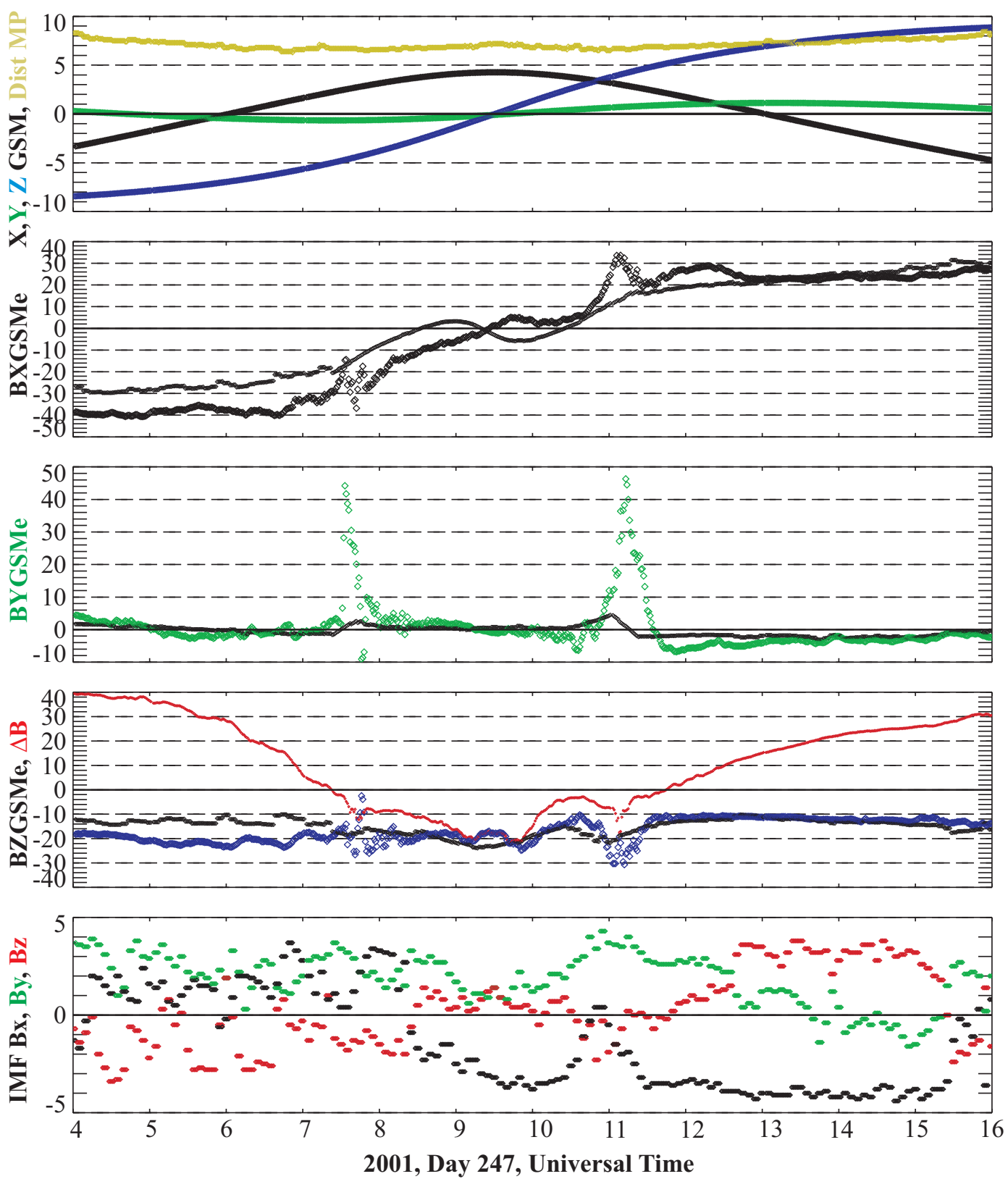

Fig. 2. An example of crossing the polar cusps by Cluster on 28 August 2001. Five panels, from top to bottom, show the spacecraft GSM position (X,Y,Z), three components of the observed (larger dots) and model (T96, smaller dots) magnetic field (only external part with the IGRF field removed; hence the "e" suffix), and three components of the concurrent IMF. Red trace in the BzGSMe panel displays the variation of the "scalar anomaly" $\Delta B=\left|\boldsymbol{B}_{\text {obs }}\right|-\left|\boldsymbol{B}_{\mathrm{IGRF}}\right|$. Note that both the Cluster $B_{y}$ spikes and the IMF $B_{y}$ have the same negative polarity in this case.

were downloaded from http://omniweb.gsfc.nasa.gov/form/ omni_min.html (King and Papitashvili, 2005).

\section{Analysis technique}

A major hurdle in the data-based studies of the polar cusps is that they are relatively narrow in latitude and, at the same time, highly dynamical. That makes it difficult to accurately predict their location, which depends not only on the seasonal/diurnal variations of the geodipole tilt angle, but also, to even greater extent, on the external conditions in the incoming solar wind and magnetosheath. In view of that, a special procedure was employed here to minimize the error in selecting the near-cusp data.

First of all, each record in the relevant yearly files of 5-min average OMNI data was provided, wherever at all possible, 


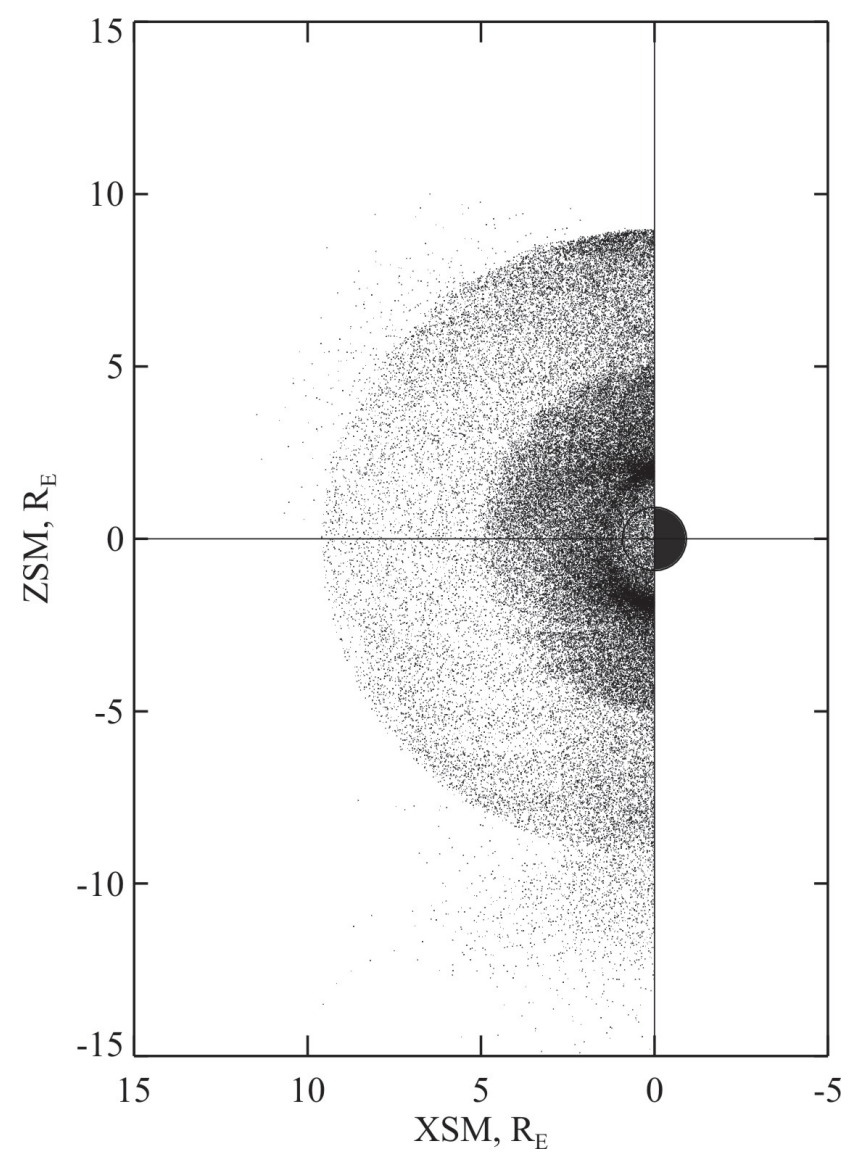

Fig. 3. Same as in Fig. 2, but for the crossing on 4 September 2001, when both IMF $B_{y}$ and the spikes of $B_{y}$ during the cusp crossings were positive.

by concurrent values of six interplanetary "driving parameters" $w_{1}, w_{2}, . ., w_{6}$ of the TS05 magnetospheric magnetic field model (Tsyganenko and Sitnov, 2005). Their derivation was performed using an automated algorithm that started with a search of prolonged quiet intervals with a steady northward IMF and then calculated sequences of the six variables $w_{i}$ as time integrals, taking into account both the interplanetary driving (via the geoeffective solar wind/IMF parameters) and individual decay timescales for each of the model field sources. (The reader is referred to the above referenced paper for more details on the model parameterization.) The relative year-by-year coverage in the combined 5-min average OMNI/TS05 yearly data files varied from $55 \%$ to $87 \%$, as shown in more detail in the following list: 1995: 68.4\% ; 1996: 63.9\%; 1997: 70.2\%; 1998: 80.1\%; 1999: 86.7\%; 2000: 66.9\%; 2001: 66.6\%; 2002: 55.3\%; 2003: $80.1 \%$; 2004: 71.1\%; 2005: 81.0\%; 2006: 79.3\%; 2007: $84.0 \%$.

At the second step, all the data were visually inspected, one day at a time, with the goal to detect and remove bad records and data taken outside the magnetosphere. As a rough filter of the magnetosheath data, the magnetopause model of Shue et al. (1998) was used, driven by the concurrent OMNI data on the solar wind pressure and IMF $B_{z}$; in addition, visual checks of the magnetopause crossings by Cluster were made with help of concurrent proton flow data of the CIS instrument, downloaded from the CDAWEB data source. Thus edited Polar and Cluster yearly data files were merged with the corresponding OMNI files, so that each data record contained all the quantities needed for the model mapping of the geomagnetic field lines. Owing to the relative dominance of the Earth's main field on the dayside, the polar cusp position approximately follows the orientation of the geodipole axis and, hence, the cusp data are better organized in the solar-magnetic (SM) coordinates rather than in the GSM system (TR99). To further narrow down the data pool for the specific purpose of this study, all data records with $X_{\mathrm{SM}}<0$ and $\left|Y_{\mathrm{SM}}\right|>5 R_{E}$ were removed.

The third step was to provide each data record with a set of parameters, allowing one to quantify the proximity of a spacecraft to the current position of the cusp field line tube. Since we have no information on the actual position of the cusp central line for any given time moment, the only possibility is to calculate it by means of the model mapping. To that end, a search of the $\min \{|\boldsymbol{B}|\}$ point at the model magnetopause was made for each data record, using its own set of solar wind/IMF and TS05 model parameters, as well as date and UT, to properly take into account the main field contribution and the geodipole tilt angle. It should be noted here that, even though the TS05 model was developed with the goal to replicate storm-time conditions, it was found to equally well reproduce quiet configurations, since all the data sets used in its construction included initial periods of undisturbed magnetosphere.

From the obtained location of the $\min \{|\boldsymbol{B}|\}$ point, a corresponding model cusp field line was traced from the magnetopause down to its ionospheric footpoint with the solarmagnetic latitude $\Lambda_{c}$ and longitude $\Phi_{c}$ (the subscript " $c$ " standing for "cusp"). Then, using a similar mapping procedure, a corresponding pair of the footpoint coordinates $\Lambda_{s c}$ and $\Phi_{s c}$ was found for the magnetic field line passing through the spacecraft location (hence the subscript "sc"). The tracing direction was towards the northern (southern) ionosphere if the spacecraft location was northward (southward) from the $\mathrm{SM}$ equatorial plane $\left(Z_{\mathrm{SM}}>0\right.$ or $Z_{\mathrm{SM}}<0$, respectively). Given the large total number of records in the Polar/Cluster data files (722714) and the complexity of the mapping procedure, it came as no surprise that the massive computation required about $40 \mathrm{~h}$ on a $3 \mathrm{GHz}$ system.

The obtained footpoint coordinates were included in each record of the output file and were then used to construct a proximity parameter, making it possible to sort the data according to their position with respect to the dynamically varying cusps and organize them into sequences of "sliding" subsets. More specifically, let us measure the footpoint SM latitude $\Lambda$ and longitude $\Phi$ in degrees and consider the following function: 
$P(\Lambda, \Phi)=\sqrt{\left(\Lambda_{c}-\Lambda\right)^{2}+\alpha^{2}\left(\Phi_{c}-\Phi\right)^{2}}$

Setting $P=P_{0}$ defines a "funnel" formed by near-cusp geomagnetic field lines, centred on the line passing through the $\min \{|\boldsymbol{B}|\}$ point at the model magnetopause, and having its footpoint at $\Lambda=\Lambda_{c}$ and $\Phi=\Phi_{c}$. The parameter $\alpha<1$ is introduced in order to control the shape of the funnel's crosssection and stretch it in the dawn-dusk direction, so that at the ionospheric level it extends over $2 P_{0}$ and $2 P_{0} / \alpha$ in latitude and longitude, respectively. Adding in (1) a variable shift $\Delta \Lambda$ makes it possible to shift the field line tube in the northsouth direction from the location of the model cusp, select the spacecraft data records that fall within each sliding funnel, and statistically analyze the obtained subsets, in particular, find those yielding the deepest field depression and the maximal correlation between the IMF $B_{y}$ and the $B_{y}$ component observed in and around the cusps. Under these assumptions, the selection criterion for a data record corresponding to a spacecraft location on a field line with footpoint at $\Lambda_{s c}, \Phi_{s c}$ can be quantified, for example, as $P\left(\Lambda_{s c}, \Phi_{s c}, \Delta \Lambda\right) \leq 1^{\circ}$, where

$P\left(\Lambda_{s c}, \Phi_{s c}, \Delta \Lambda\right)=\sqrt{\left(\Lambda_{c} \pm \Delta \Lambda-\Lambda_{s c}\right)^{2}+\alpha^{2}\left(\Phi_{c}-\Phi_{s c}\right)^{2}}$

in which case the sliding funnel covers $2^{\circ}$ in latitude and $2^{\circ} / \alpha$ in longitude at its base (i.e., at the ionospheric level). The plus (minus) sign before $\Delta \Lambda$ in Eq. (2) is chosen if the spacecraft and the model cusp are in the Northern (Southern) Hemisphere, which ensures that in both cases (i.e., in both Northern and Southern Hemispheres), positive (negative) values of $\Delta \Lambda$ result in a poleward (equatorward) shift of the selection funnel.

\section{Results}

The cusp structure was analyzed by creating 201 subsets of data records from the above described cumulative set, corresponding to the sequence of 201 consecutive values of the shift parameter $\Delta \Lambda=-10^{\circ}+0.1^{\circ}(i-1)(i=1,2, \ldots, 201)$. Accordingly, the selection funnel spanned the interval within $\pm 10^{\circ}$ around the footpoint latitude of the cusp central line, predicted by the TS05 model individually for each magnetic field data record in the cumulative set. Figure 4 illustrates the spatial distribution in solar-magnetic coordinates of the data points selected by this procedure. Left side panels show all the data that contributed in the 201 subsets, and the right side ones correspond to only one subset with $\Delta \Lambda=0$. Each subset was further divided into ten $1 R_{E}$ bins of the radial distance in the range $2 \leq R \leq 12 R_{E}$ and, for each bin, we calculated (a) the average value and the rms scatter of the difference $\Delta B=\left|\boldsymbol{B}_{\text {obs }}\right|-\left|\boldsymbol{B}_{\mathrm{IGRF}}\right|$ between the magnitudes of the total observed field and of the Earth's main field, (b) correlation coefficients between the $B_{y}$ field component observed in and around the cusp and $B_{y}^{(\mathrm{IMF})}$, and (c) parameters $C_{1}$ and $C_{2}$ in the linear regression equation

$B_{y}=C_{1} B_{y}^{(\mathrm{IMF})}+C_{2}$

Figure 5 shows a set of 10 plots of the correlation coefficient (c.c.) and of $\Delta B$ as functions of $\Delta \Lambda$, corresponding to 10 intervals of radial distance $R$, from the closest one (top left) to the most distant (bottom right). In each bin of $R$, one clearly sees a peak of the c.c. at the level of $\sim 0.6$, located about $\Delta \Lambda \sim 3^{\circ}$ poleward from the model cusp footpoint. The peaks are relatively sharper at $R \leq 7 R_{E}$ and become more spread out in the outer cusp and magnetosheath at $R \geq 8 R_{E}$. The plots of $\Delta B$, in contrast to those for the c.c., are virtually flat at $R \leq 5$, and the first signs of the cusp diamagnetism become visible at $5 \leq R \leq 6$ as a broad and shallow field depression. In agreement with a simple estimate based on the pressure balance across the cusp (TR99), the depression rapidly deepens with further growing $R$. Its peak values reach $\sim-40 \mathrm{nT}$ at $8 \leq R \leq 9$, and then eventually subside as one enters in the outermost cusp "throat", opening into the magnetosheath. Also note that, in comparison with the c.c. peaks, the peak values of $\Delta B$ are located at $\Delta \Lambda \sim 2^{\circ}$, that is, closer by $\sim 1^{\circ}$ to the model cusp centre.

An important factor controlling the exposure of the polar cusps to the incoming solar wind is the geodipole tilt angle $\Psi$. Therefore, one may expect the above effects to be strongly modulated by that parameter. To explore its role in more quantitative detail, two separate subsets of near-cusp data were created, corresponding to small and large angles of incidence of the solar wind upon the cusp "throat". For the sake of brevity, these two cases will be referred to below as S- and W-cases, respectively, since they are typical for summer and winter conditions. Specifically, the S- (W-) subsets included (1) the data taken in the vicinity of the northern (southern) cusp during the periods with $\Psi \geq 15^{\circ}$ and (2) the data from the southern (northern) cusp with $\Psi \leq-15^{\circ}$. The obtained subsets were then used in the same computational procedure as described above, with the results for the S- and W-cases being shown in Figs. 6 and 7, respectively, in the same format as in Fig. 5.

The first thing that immediately stands out in Fig. 6, in comparison with the "all-season" case shown in Fig. 5, is strikingly higher peak values of the c.c., reaching 0.93 for the radial bin $4 \geq R \geq 5$. Also, the poleward shifts of the c.c. peaks from the model cusp are somewhat smaller in all radial bins, while all the peaks are generally wider in latitude, implying a stronger "splitting" by the penetrating plasma due to the smaller incidence angle and, hence, more effective exposure to the incoming plasma flow. The field depression also drops to lower values, with the largest effect observed in the $7 \leq R \leq 8$ bin, where $\Delta B$ reaches $\approx-53 \mathrm{nT}$.

In the opposite case of the least exposed cusp (W-case), shown in Fig. 7, all the above effects are much weaker and less regular than in the previous S-case. As already said, the main reason is the "more leeward" location of the winter 

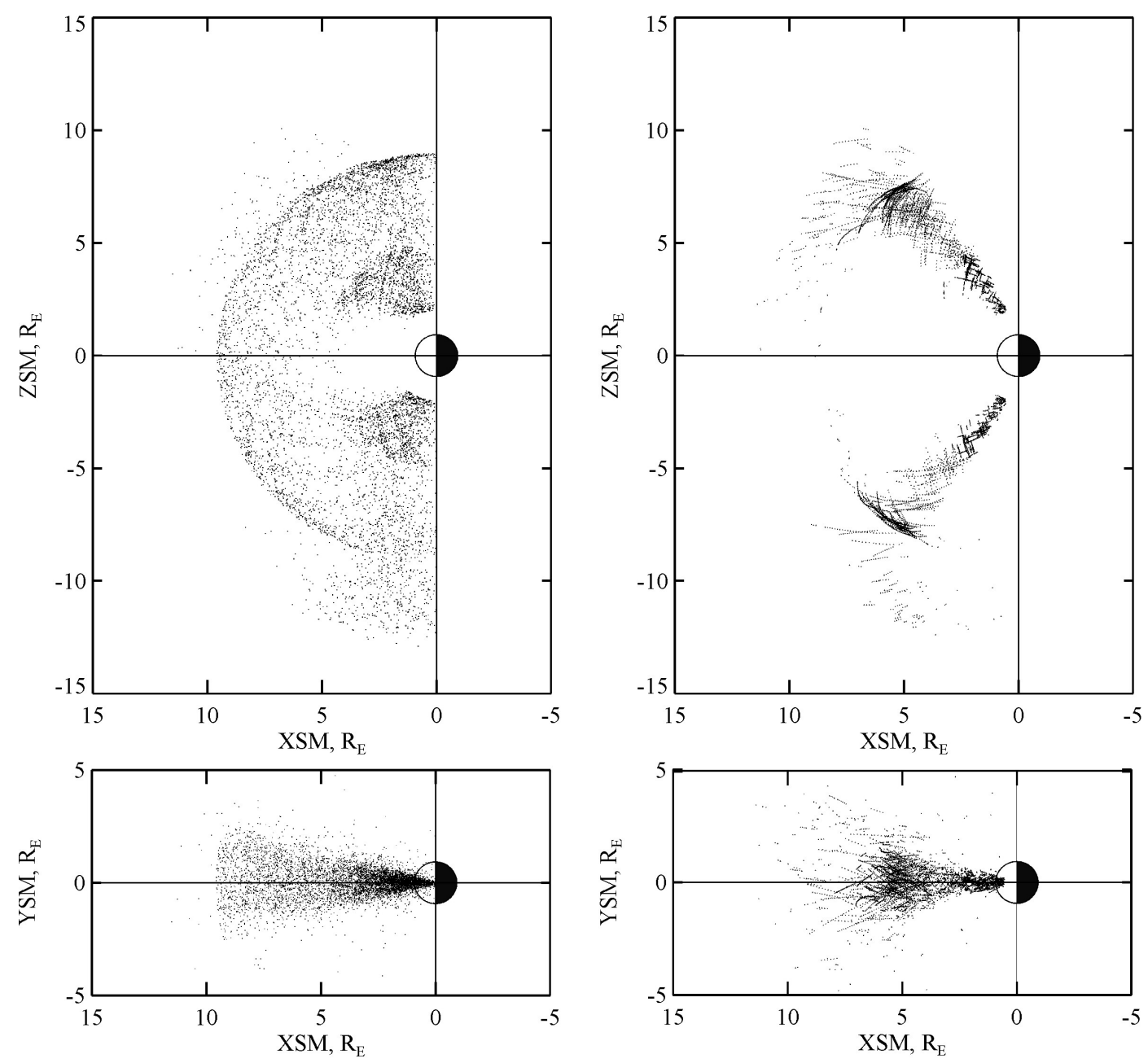

Fig. 4. Distribution of Polar/Cluster near-cusp data obtained using the "sliding funnel" method, in projection on the XSM-ZSM (above) and XSM-YSM (below) planes. Left panels: cumulative set of data points corresponding to all 201 values of the footpoint latitude shift in the range $-10^{\circ} \leq \Delta \Lambda \leq+10^{\circ}$ (total number of records $N=72516$ ). Right panels: a subset of the cumulative set, corresponding to a single value $\Delta \Lambda=0$ (total number of records $N=5732$ ).

cusps; another factor is a much poorer coverage by data in this case, especially in the outermost high-latitude bins of the radial distance $\left(R>9 R_{E}\right)$, located beyond Polar's apogee and seldom visited by Cluster during winter months. (Gaps in the plots correspond to sliding subsets with less than 20 data records).

To more accurately establish the relative role of the main factors controlling the magnitude of the cusp field depression $\Delta B$, it was represented as a linear function of the dipole tilt $\Psi$, solar wind ram pressure $P_{\mathrm{dyn}}$, and $B_{z}^{(\mathrm{IMF})}$ :

$\Delta B=a_{1} \Psi+a_{2} P_{\mathrm{dyn}}+a_{3} B_{z}^{(\mathrm{IMF})}+a_{4}$

and a trilinear regression fit was carried out for the same set of data subsets that contributed to the above results, illustrated in Fig. 5. The main conclusions drawn from that analysis are as follows. (a) As expected, the first two controlling variables, $\Psi$ and $P_{\text {dyn }}$ are the dominant factors affecting the depth of the cusp diamagnetic depression. (b) The third variable, $B_{z}^{(\mathrm{IMF})}$, plays virtually no role here. As an example, in the radial bin $6 \leq R \leq 7 R_{E}$, the values of the regression coefficients in Eq. (4), corresponding to the negative peak value of $\Delta B=-28.6 \mathrm{nT}$, were found $a_{1}=-35.6 \pm 3.4$, $a_{2}=-13.4 \pm 0.4, a_{3}=-0.06 \pm 0.24, a_{4}=5.5 \pm 1.3$, and the correlation between the regression model (4) and the corresponding data subset was 0.89 .

Based on this result, one can see that the amplitude of the depression variation between summer and winter conditions (with the tilt angle $\Psi$ varying between -0.6 and +0.6 radians) can be as large as $40 \mathrm{nT}$, and roughly the same range of variation will result from the wind ram pressure varying between $2 \mathrm{nPa}$ (average quiet) and $5 \mathrm{nPa}$. At the same time, variation of $B_{z}^{(\mathrm{IMF})}$ within $\pm 5 \mathrm{nT}$ (corresponding to $\sim 90 \%$ of all cases) contributes less than $1 \mathrm{nT}$ to $\Delta B$, in agreement with our earlier qualitative result (TR99). 

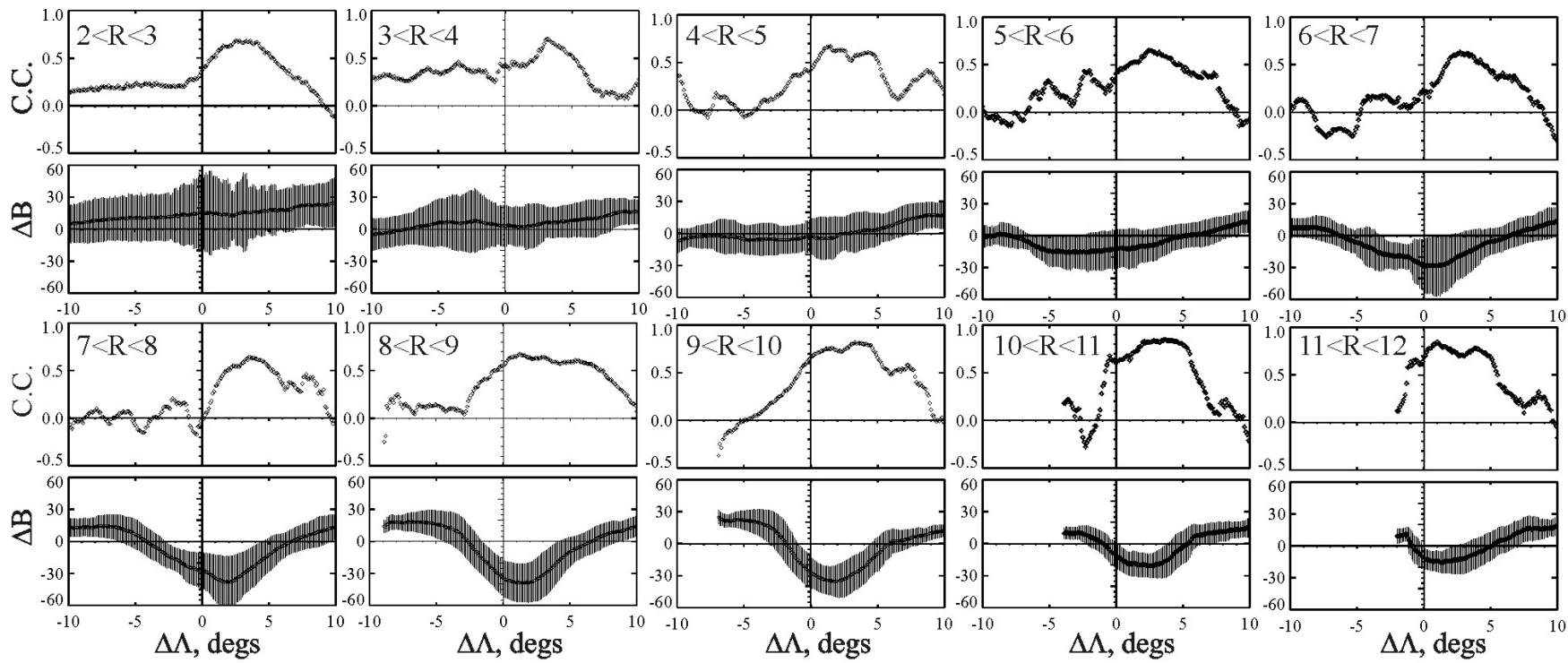

Fig. 5. Latitudinal profiles across the model cusp of the correlation coefficient between $B_{y}$ field component observed in and around the cusp and $B_{y}^{(\mathrm{IMF})}$ (upper panels in each of the 10 plots), and of the scalar anomaly $\Delta B=\left|\boldsymbol{B}_{\text {obs }}\right|-\left|\boldsymbol{B}_{\text {IGRF }}\right|$ (lower panels). Both quantities are plotted for 10 intervals of the radial distance $R$, covering the range $2 \leq R \leq 12 R_{E}$. Rms deviation of $\Delta B$ is shown by vertical shading.
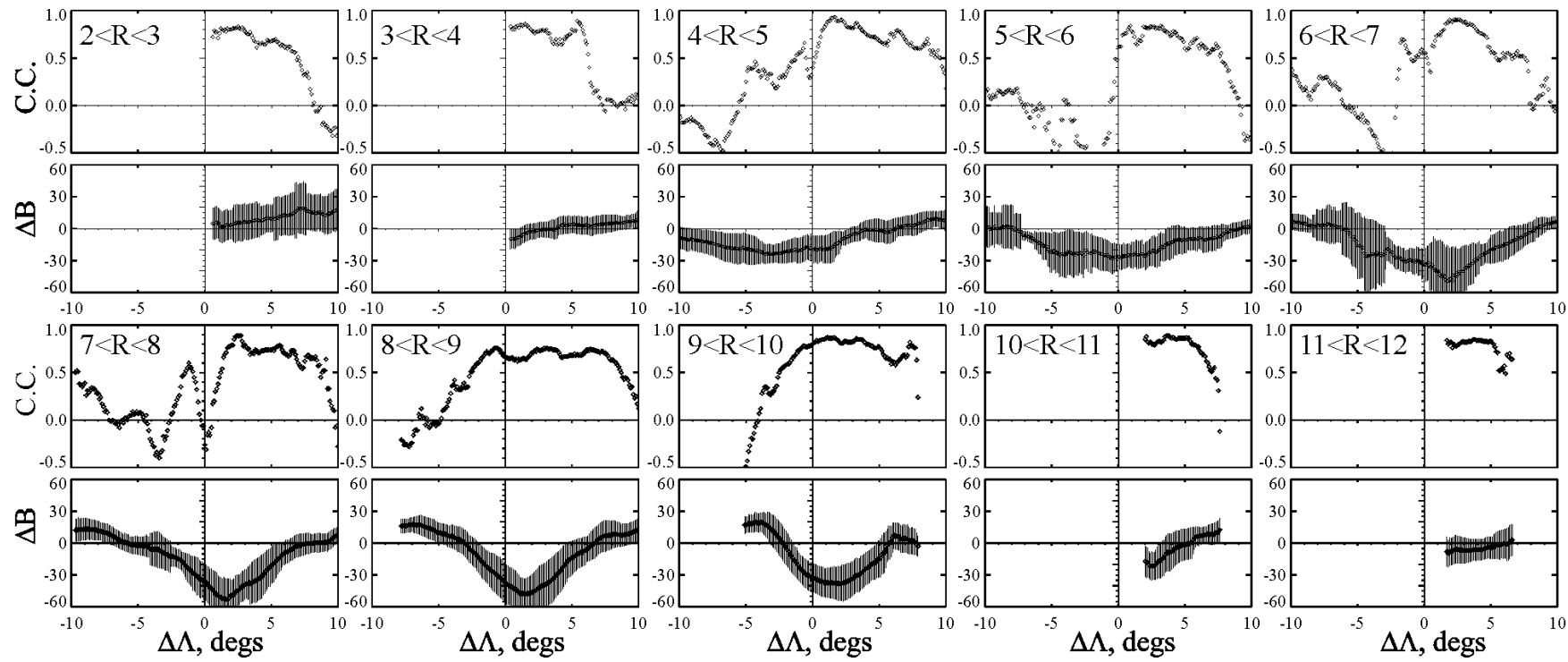

Fig. 6. Same as in Fig. 5, but for the maximal exposure of the polar cusps to the incoming solar wind: $\Psi \geq+15^{\circ}$ and $\Psi \leq-15^{\circ}$ for the northern and southern polar cusps, respectively. (The "summertime" case S.)

\section{Discussion: IMF $B_{y}$ "penetration" and field-aligned cusp currents}

Figure 8 displays a set of 10 latitudinal profiles of the coefficient $C_{1}$ in the regression Eq. (3), corresponding to the same 10 intervals of the radial distance as in the previous plots. The coefficient quantifies the effect of amplification of the IMF $B_{y}$ inside the cusps, and a remarkable feature clearly seen in the plots is its regular variation between the consecutive radial distance bins. The largest amplification rate, $C_{1} \approx 12.6$, is observed in the closest bin $2 \leq R \leq 3$ at $\Delta \Lambda=3.4^{\circ}$, then it rapidly falls off with growing $R$ and reaches the minimal peak value $C_{1} \approx 2.4$ in the radial bin $6 \leq R \leq 7$ at $\Delta \Lambda \approx 2.5^{\circ}$. Beyond that distance the trend reverses, and the coefficient gradually increases to its asymptotic value $C_{1} \approx 5.2$ at $\Delta \Lambda=1.0^{\circ}$ in the outermost bin of $R$.

The steep decrease of $C_{1}$ along the cusp can be naturally interpreted as due to a pair of oppositely directed 


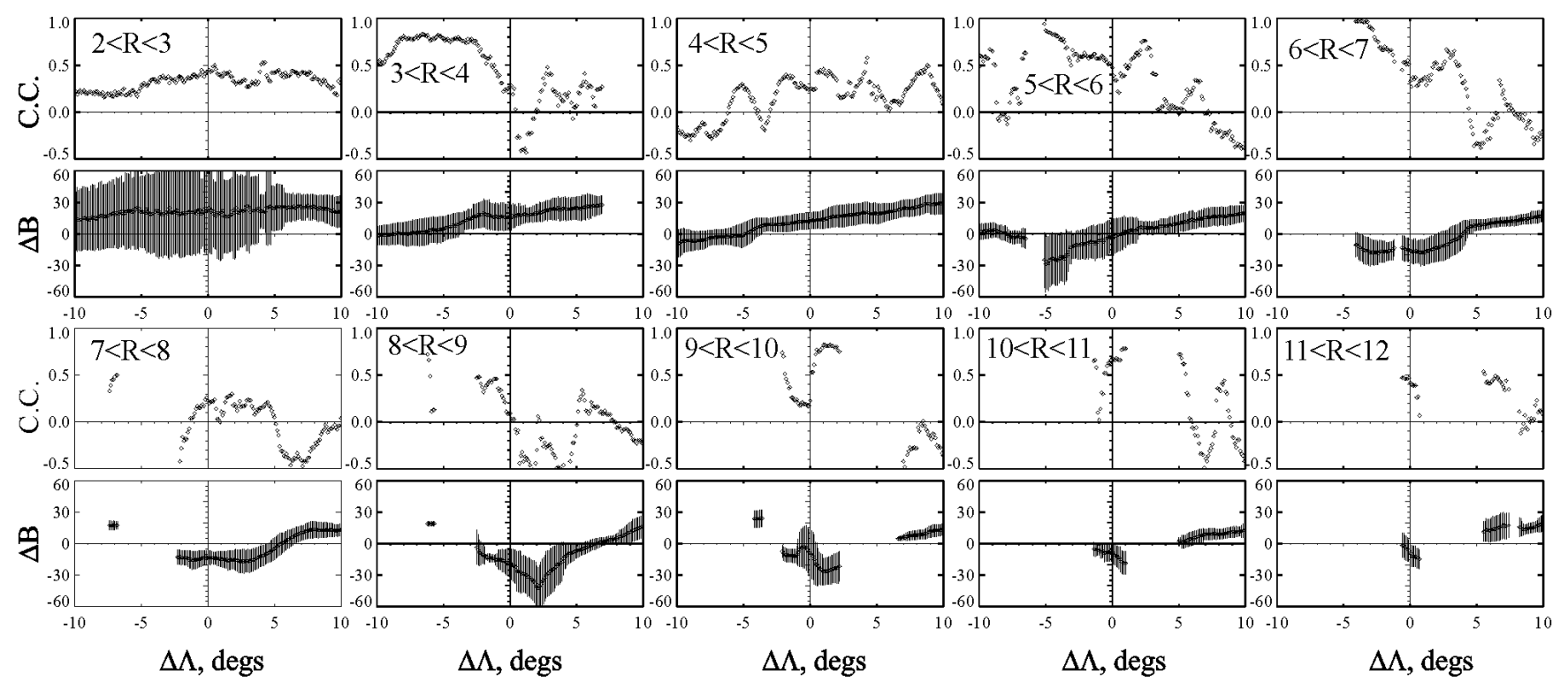

Fig. 7. Same as in Fig. 6, but for the minimal exposure of the polar cusps to the incoming solar wind: $\Psi \leq-15^{\circ}$ and $\Psi \geq+15^{\circ}$ for the northern and southern polar cusps, respectively. (The "wintertime" case W.)

field-aligned currents near the noon meridian: for positive (dawn-dusk) IMF $B_{y}$, the downward current flows a few degrees equatorward from the field line corresponding to the peak of $C_{1}$ and the upward current is located poleward from that line; in the case of negative IMF $B_{y}$ the field-aligned currents have the opposite polarity. As already noted in the Introduction, this interpretation is by no means new: the positive correlation of near-cusp $B_{y}$ with IMF $B_{y}$ and its association with field-aligned currents was reported three decades ago by McDiarmid et al. $(1978,1979)$ from observations onboard ISIS-2 spacecraft. That finding was confirmed in a later work by Erlandson et al. (1988) based on measurements onboard Viking, in which the near-cusp magnetic effects of IMF $B_{y}$ were explained as a result of longitudinal shift of the near-noon Region 1 and higher-latitude midday cusp currents, also termed as Region 0 (Ohtani et al., 1995). In a more recent paper (Taguchi et al., 1993) based on DE-2 data, the effect was reconfirmed but, unlike in the previous studies, its interpretation was made in terms of a detached highlatitude pair of sheets of field-aligned currents, entirely separate from the Region 1 system and induced by the joint effect of the dayside reconnection and the magnetosheath plasma flow. The next leap forward was made owing to the launch of Polar in 1996, whose observations revealed new interesting details of the cusp structure; in particular, the magnitude and spatial extent of the diamagnetic depression were analyzed in TR99, while the IMF $B_{y}$ impact on the dayside field-aligned currents was studied by Zhou et al. (2000); both works used only Northern Hemisphere data taken in 1996 and 1997.

The present work, to our knowledge, is the first systematic study of the above issue, based on data from the entire range of distances between 2 and $12 R_{E}$, and covering both northern and southern cusps. Getting back to the obtained radial variation of the coefficient $C_{1}$, shown in Fig. 8, note that it can be used as a simple quantitative test of the proposed interpretation of the IMF $B_{y}$ "penetration" in the cusps as due to the effect of the pair of oppositely directed field-aligned currents. Indeed, the assumption $\boldsymbol{j} \| \boldsymbol{B}$ with $B \sim R^{-3}$ (which holds at $R \leq 6 R_{E}$ ) implies that the associated transverse magnetic disturbance varies as $\sim R^{-\gamma}$, where $\gamma \approx 3 / 2$ (e.g., Tsyganenko, 2002). This is reasonably close to what follows from the above plot: calculating the ratios of the peak magnitudes $C_{1}^{(i)}$ for four consecutive radial bins, centred at $R_{i}=2.5,3.5,4.5$, and $5.5(i=1,2,3,4)$ we calculate the corresponding $\gamma_{i}$ as $\ln \left(C_{1}^{(i+1)} / C_{1}^{(i)}\right) / \ln \left(R_{i+1} / R_{i}\right)$, with the following result: $\gamma_{1}=1.35, \gamma_{2}=2.15, \gamma_{3}=1.53, \gamma_{4}=2.14$, which roughly agrees with the above estimate.

The observed reversal of $\partial C_{1} / \partial R$ from negative to positive and the increase of $C_{1}$ with growing distance beyond $R=6-7 R_{E}$, with an apparent saturation at the level $B_{y} / B_{y}^{\mathrm{IMF}} \approx 5.2$ in the outermost distance bin (panels for $10 \leq R \leq 11$ and $11 \leq R \leq 12$ in Fig. 8), is a result of a gradual opening of the cusp throat into the magnetosheath. Because of the compression of the magnetic field and plasma on crossing the bow shock, the magnetic field typically rises there by the factor of $\sim 3$ from its undisturbed values in the upstream solar wind (e.g., Zhuang and Russell, 1981) and, due to the pile-up effect in the magnetosheath, it further rises toward the magnetopause by the factor $\sim 1.5-2.0$ (Hill et al., 1995). The resultant total amplification of the IMF amounts to the factor 5-6 (see also a review by Fairfield, 1976), which is what one sees in Fig. 8. 

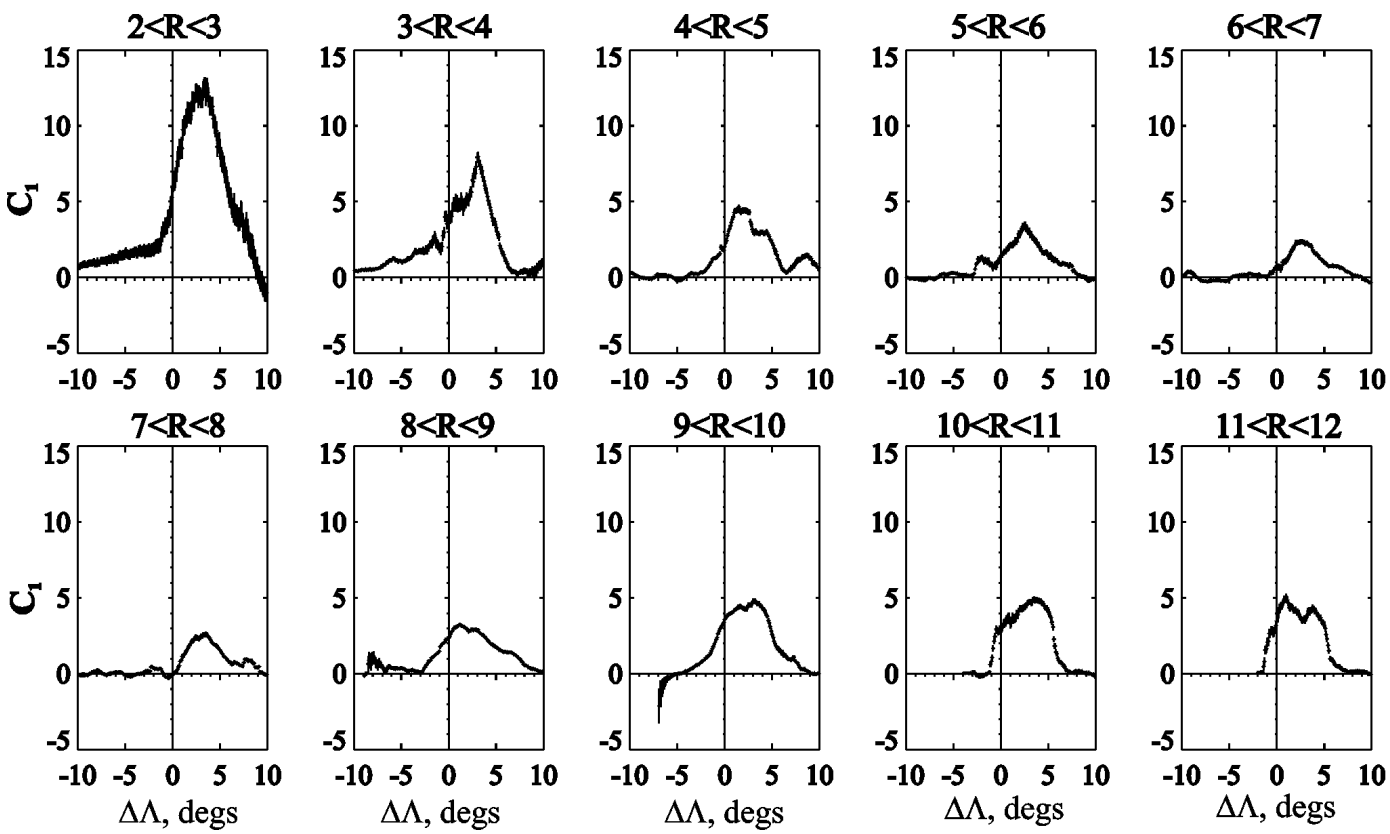

Fig. 8. Latitudinal profiles across the model cusp of the first regression coefficient $C_{1}$ of proportionality between the $B_{y}$ field component observed in and around the cusp and $B_{y}^{(\mathrm{IMF})}$, for 10 intervals of the radial distance $R$. Note a regular variation of the peak values of $C_{1}$ with growing radial distance, consistent with the model of field-aligned cusp currents, responsible for the observed "penetration" of the IMF $B_{y}$.

A persistent feature that sticks out in all the above plots is the systematic poleward shift with respect to the model polar cusp field line of the peaks of the c.c. and $\Delta B$ (Figs. 5-6), and of the regression coefficient $C_{1}$ (Fig. 8). Let us consider first the shift in $\Delta B$ plots. Intuitively, one might expect the peaks of the cusp diamagnetic depression $\Delta B$ to be colocated with the midday field line that passes through the location of $\min \{|\boldsymbol{B}|\}$ at the magnetopause and serves as a dividing line between the dayside and tail lobe field lines. However, our calculations indicate that the actually observed depressions map about $2^{\circ}$ poleward from that dividing line. Two factors could have contributed to the obtained shift. The first one is the nearly axisymmetric shape of the model magnetopause, assumed in the TS05 model as a fit to the empirical boundary of Shue et al. (1998). Even though the assumption of the axial symmetry can be fairly accurate on a large scale, it is definitely not so in the high-latitude dayside region, where the actual boundary may have deep indentations required by the pressure balance condition (Sotirelis and Meng, 1999; Boardsen et al., 2000; however, see an alternate view in Zhou and Russell, 1997). The second factor is that the actual physics of the cusp formation is much more complex than in idealized models: in particular, neither TS05 nor any other empirical model take into account magnetic effects associated with the cusp plasma. In this regard, note that a similar small poleward shift $\left(1-2^{\circ}\right.$ of the footpoint latitude) of the observed cusp from that predicted by the T96 model was also reported by Zhou et al. (1999).
A somewhat larger shift (about $3^{\circ}$ ) in the c.c. and $C_{1}$ plots is easier to understand, given the fact that both quantities refer to the $B_{y}$ disturbance that peaks between two fieldaligned current sheets, located on the poleward side of the cusp. According to Erlandson et al. (1988), the equatorward current coincides with the cusp and the poleward one flows in the mantle (see their Fig. 10), while Taguchi et al. (1993) placed both current sheets at even higher latitudes, as a pair of currents entirely separate from the cusp (their Fig. 7).

\section{Conclusions}

A detailed study was carried out of the magnetic structure of the polar cusps, using multi-year sets of Polar and Cluster data, taken during 1996-2006 and 2001-2007, respectively, and covering the entire length of both northern and southern cusps, from geocentric $\left(R \sim 2 R_{E}\right)$ to the cusp "throat" $\left(R \sim 12-13 R_{E}\right)$. All the cusp data were selected and sorted into "sliding funnel" subsets, using magnetic field models in order to properly take into account the dipole tilt and solar wind/IMF effects, and each subset was further divided into 10 bins of the radial distance. A linear regression analysis of the binned data revealed the following facts. (1) Diamagnetic depression, associated with the magnetosheath plasma injected in the cusps, becomes first visible at $R \sim 4-5 R_{E}$, then rapidly deepens with growing $R$, maximizes at $R \sim 6-9 R_{E}$, and partially subsides and widens in latitude at the cusp's outermost end. (2) The depression peak is systematically shifted 
poleward (by $\sim 2^{\circ}$ in terms of the footpoint latitude) with respect to the model cusp field line. (3) At all radial distances, clear and distinct peaks of the correlation between the local $B_{y}$ and $B_{y}^{(\mathrm{IMF})}$, and of the corresponding regression scaling coefficient $C_{1}$ are observed with a remarkably regular variation of the latter coefficient with the radial distance, which quantitatively confirms the previously proposed concept of the cusp field-aligned currents.

Acknowledgements. It is a pleasure to acknowledge the teams and PIs of all experiments whose data were used in this study. The data of Polar MGF experiment were made available online by the UCLA team led by the PI C. T. Russell. The Cluster magnetometer, spacecraft ephemeris, and CIS instrument data were obtained from the NSSDC CDAWEB online facility (originally provided by the PIs: A. Balogh, M. Tatrallyay, and H. Rème, respectively). HighResolution OMNI interplanetary data were obtained from the SPDF OMNIWEB interface (R. McGuire, N. Papitashvili). This work was supported by the RFBR grants 07-02-91703 and 07-05-91109.

Topical Editor I. A. Daglis thanks G. Siscoe and another anonymous referee for their help in evaluating this paper.

\section{References}

Balogh, A., Dunlop, M. W., Cowley, S. W. H., Southwood, D. J., Thomlinson, J. G., Glassmeier, K. H., Musmann, G., Lühr, H., Buchert, S., Acuna, M. H., Fairfield D. H., Slavin, J. A., Riedler, W., Schwingenschuh, K., and Kivelson, M. G.: The Cluster magnetic field investigation, Space Sci. Rev., 79, 65-92, 1997.

Boardsen, S. A., Eastman, T. E., Sotirelis, T., and Green, J. L.: An empirical model of the high-latitude magnetopause, J. Geophys. Res., 105, 23193-23219, 2000.

Chapman, S. and Ferraro, V. C. A.: A new theory of magnetic storms, J. Geophys. Res., 36, 171-186, 1931.

Erlandson, R. E., Zanetti, L. J., Potemra, T. A., Bythrow, P. F., and Lundin, R.: IMF By dependence of Region 1 Birkeland currents near noon, J. Geophys. Res., 93, 9804-9814, 1988.

Fairfield, D. H.: Magnetic Fields of the Magnetosheath, Rev. Geophys. Space Phys., 14, 117-134, 1976.

Hill, P., Paschmann, G., Treumann, R. A., Baumjohann, W., Sckopke, N., and Lühr, H.: Plasma and magnetic field behavior across the magnetosheath near local noon, J. Geophys. Res., 100, 9575-9583, 1995.

King, J. H. and Papitashvili, N. E.: Solar wind spatial scales in and comparisons of hourly Wind and ACE plasma and magnetic field data, J. Geophys. Res., 110, A02104, doi:10.1029/2004JA010649, 2005.
McDiarmid, I. B., Burrows, J. R., and Wilson, M. D.: Magnetic Field Perturbations in the Dayside Cleft and Their Relationship to the IMF, J. Geophys. Res., 83, 5753-5756, 1978.

McDiarmid, I. B., Burrows, J. R., J. R., and Wilson, M. D.: LargeScale Magnetic Field Perturbations and particle measurements at $1400 \mathrm{~km}$ on the dayside, J. Geophys. Res., 84, 1431-1441, 1979.

Ohtani, S., Potemra, T. A., Newell, P. T., Zanetti, L. J., Iijima, T., Watanabe, M., Yamauchi, M., Elphinstone, R. D., de la Beaujardiere, O., and Blomberg, L. G.: Simultaneous prenoon and postnoon observations of three field-aligned current systems from Viking and DMSP-F7, J. Geophys. Res., 100, 119-136, 1995.

Russell, C. T., Snare, R. C., Means, J. D., Pierce, D., Dearborn, D., Larson, M., Barr, G., and Le, G.: The GGS/Polar magnetic fields investigation, Space Sci. Rev., 71, 563-582, 1995.

Shue, J.-H., Song, P., Russell, C. T., Steinberg, J. T., Chao, J. K., Zastenker, G., Vaisberg, O. L., Kokubun, S., Singer, H. J., Detman, T. R., and Kawano, H.: Magnetopause location under extreme solar wind conditions, J. Geophys. Res., 103, 1769117710, 1998.

Sotirelis, T. and Meng, C.-I.: Magnetopause from pressure balance, J. Geophys. Res., 104, 6889-6898, 1999.

Tsyganenko, N. A. and Russell, C. T.: Magnetic signatures of the distant polar cusps: Observations by Polar and quantitative modeling, J. Geophys. Res., 104, 24939-24955, 1999.

Tsyganenko, N. A.: A model of the near magnetosphere with a dawn-dusk asymmetry: 1. Mathematical structure, J. Geophys. Res., 107(A8), 1179, doi:10.1029/2001JA000219, 2002.

Tsyganenko, N. A. and Sitnov, M. I.: Modeling the dynamics of the inner magnetosphere during strong geomagnetic storms, J. Geophys. Res., 110, A03208, doi:10.1029/2004JA010798, 2005.

Zhang, H., Dunlop, M. W., Zong, Q.-G., Fritz, T. A., Balogh, A., and Wang, Y.: Geometry of the high-latitude magnetopause as observed by Cluster, J. Geophys. Res., 112, A02204, doi:10.1029/2006JA011774, 2007.

Zhou, X.-W. and Russell, C. T.: The location of the high-latitude polar cusp and the shape of the surrounding magnetopause, J. Geophys. Res., 102, 105-110, 1997.

Zhou, X.-W., Russell, C. T., Le, G., Fuselier, S. A., and Scudder, J. D.: The polar cusp location and its dependence on dipole tilt, Geophys. Res. Lett., 26, 429-432, 1999.

Zhou, X.-W., Russell, C. T., and Le, G.: Local time and interplanetary magnetic field By dependence of field-aligned currents at high altitudes, J. Geophys. Res., 105, 2533-2539, 2000.

Zhuang, H. C. and Russell, C. T.: An analytic treatment of the structure of the bow shock and magnetosheath, J. Geophys. Res., 86, 2191-2205, 1981. 